\title{
ARTICLE
}

\section{A diet enriched with curcumin promotes resilience to chronic social defeat stress}

\author{
Antonio V. Aubry ${ }^{1,2}$, Hameda Khandaker ${ }^{1,3}$, Rebecca Ravenelle ${ }^{1,4}$, Itamar S. Grunfeld ${ }^{1,3}$, Valentina Bonnefil ${ }^{5}$, Kenny L. Chan ${ }^{2}$, \\ Flurin Cathomas ${ }^{2}$, Jia Liu ${ }^{5}$, Glenn E. Schafe ${ }^{1,3}$ and Nesha S. Burghardt ${ }^{1,3}$
}

\begin{abstract}
Chronic exposure to stress is a well-known risk factor for the development of mood and anxiety disorders. Promoting resilience to stress may prevent the development of these disorders, but resilience-enhancing compounds are not yet clinically available. One compound that has shown promise in the clinical setting is curcumin, a polyphenol compound found in the rhizome of the turmeric plant (Curcuma longa) with known anti-inflammatory and antidepressant properties. Here, we tested the efficacy of $1.5 \%$ dietary curcumin at promoting resilience to chronic social defeat stress (CSDS) in 129/SvEv mice, a strain that we show is highly susceptible to this type of stress. We found that administration of curcumin during CSDS produced a 4.5-fold increase in stress resilience, as measured by the social interaction test. Although the overall effects of curcumin were striking, we identified two distinct responses to curcumin. While $64 \%$ of defeated mice on curcumin were resilient (responders), the remaining $36 \%$ of mice were susceptible to the effects of stress (non-responders). Interestingly, responders released less corticosterone following acute restraint stress and had lower levels of peripheral IL-6 than nonresponders, implicating a role for the NF-KB pathway in treatment response. Importantly, curcumin also prevented anxiety-like behavior in both responders and non-responders in the elevated-plus maze and open field test. Collectively, our findings provide the first preclinical evidence that curcumin promotes resilience to CSDS and suggest that curcumin may prevent the emergence of a range of anxiety-like symptoms when given to individuals during exposure to chronic social stress.
\end{abstract}

Neuropsychopharmacology (2019) 44:733-742; https://doi.org/10.1038/s41386-018-0295-2

\section{INTRODUCTION}

Mood and anxiety disorders are among the most prevalent of all psychiatric disorders [1]. Numerous risk factors have been identified for developing these disorders, with chronic stress being the best characterized [2, 3]. Interestingly, not everyone exposed to chronic stress develops a mood or anxiety disorder. This observation has triggered interest in understanding the mechanisms that underlie individual differences in response to stress and the development of strategies to promote stress resilience. Advances have been made in preclinical studies using chronic social defeat stress (CSDS), which is an ethologically relevant rodent model used to characterize mice as either "resilient" or "susceptible" to stress based on social avoidance behavior. This social avoidance phenotype has face validity in modeling avoidance behavior seen in patients with PTSD and social withdrawal commonly reported in depressed patients. Findings from CSDS studies indicate that stress susceptibility and resilience are each associated with distinct changes in signal transduction pathways, gene transcription, and neurophysiological activity across cortico-limbic circuits [4-6]. In the periphery, susceptible and resilient animals also display distinct inflammatory and metabolic responses to stress [7-9]. One approach for promoting stress resilience has been to block mechanisms implicated in susceptibility using optogenetics or overexpression of transcription factors $[10,11]$. However, this work has not yet led to effective treatments in the clinic.

One treatment that has shown promise in the clinical setting is curcumin. Curcumin is a polyphenol compound found in the rhizome of the turmeric plant (Curcuma longa) with known chemopreventative, neuroprotective, and anti-inflammatory properties ([12-14]. It has been used to treat several diseases with an inflammatory component, including rheumatoid arthritis, ulcerative colitis, and diabetes [15-18]. Interestingly, curcumin may also be effective in treating psychiatric disorders, such as major depressive disorder. Recent double-blind placebo-controlled studies indicate that the effects of curcumin are particularly pronounced in patients with atypical depression, which is associated with somatic symptoms and high levels of inflammatory cytokines $[19,20]$. Similarly, preclinical studies show that curcumin administered during exposure to chronic stress in rodents reduces the development of depressive-like behavior and prevents stress-induced morphological and functional changes in the hippocampus $[13,21,22]$.

Although there is growing interest in the use of curcumin for treating psychiatric disorders, to our knowledge no studies have

\footnotetext{
${ }^{1}$ Department of Psychology, Hunter College, The City University of New York, New York, NY, USA; ${ }^{2}$ Department of Neuroscience and Friedman Brain Institute, Icahn School of Medicine at Mount Sinai, New York, NY, USA; ${ }^{3}$ Department of Psychology, The Graduate Center, The City University of New York, New York, NY, USA; ${ }^{4}$ Department of Biology, The

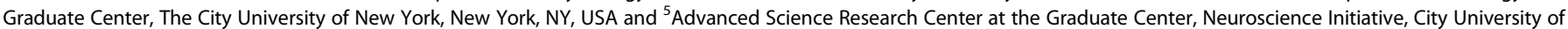
New York, New York, NY, USA

Correspondence: Nesha S. Burghardt (nb844@hunter.cuny.edu)
}

Received: 17 July 2018 Revised: 19 November 2018 Accepted: 27 November 2018

Published online: 12 December 2018 
734

investigated whether curcumin promotes resilience against social defeat stress or prevents the emergence of symptoms of anxiety that frequently follow periods of chronic stress. Accordingly, we administered dietary curcumin to mice during CSDS and characterized mice as resilient or susceptible based on their social avoidance behavior in the social interaction test. Experiments were conducted in $129 / \mathrm{SvEv}$ mice, a strain that we show is highly susceptible to this type of stress. We found that curcumin leads to a 4.5-fold increase in the proportion of resilient mice. Resilient mice had lower levels of plasma IL-6 and their HPA axis response to a novel stressor was attenuated compared to susceptible mice fed curcumin. Curcumin also protected against stress-induced increases in anxiety-like behavior in the elevated-plus maze and open field test. Our findings suggest that curcumin may safely and effectively be used to prevent social avoidance and other symptoms of anxiety when it is administered to individuals during exposure to chronic social stress.

\section{MATERIALS AND METHODS}

Animals

Male 129/SvEv (Taconic Biosciences, Germantown, NY) and C57BL/6J mice (Jackson Laboratory, Bar Harbor, ME) were purchased at 8 weeks of age and group housed (4/cage) upon arrival with mice of the same strain. Retired male CD-1 breeders were purchased from Charles River Laboratories (Wilmington, MA) and individually housed upon arrival. Mice acclimated to the colony room for a minimum of 1 week and were maintained on a $12 \mathrm{~h}$ (05:00-17:00) light-dark schedule with free access to food and water. Experiments were conducted in accordance with $\mathrm{NIH}$ guidelines and were approved by the Institutional Animal Care and Use Committee of Hunter College.

\section{Curcumin diet}

Five days prior to social defeat stress, standard laboratory chow was replaced with either a global $18 \%$ protein chow diet (Control chow; Envigo Teklad) or a global $18 \%$ protein chow diet commercially made with $1.5 \%$ curcumin (Curcumin chow; Pfaltz \& Bauer, $95 \%$ diferuloylmethane), both of which were provided in pellet form. Mice remained on their respective diets throughout the duration of the experiment. This protocol was based on two previous studies, one of which showed that dietary curcumin impairs the formation of fear memories when it is given 5 days before training and throughout behavioral testing [23]. Another study demonstrated that oral curcumin blocks stress-induced deficits in spatial memory when it is administered during exposure to chronic restraint stress [13]. We also performed a separate experiment, in which administration of curcumin or control chow began after social defeat stress. A high concentration of curcumin was chosen based on the known low bioavailability of the compound [24]. We found that mice fed either diet consumed approximately $3-5 \mathrm{~g}$ of food per day ( $45-75 \mathrm{mg}$ curcumin/day) and did not differ in average daily food intake (control chow: $4.02 \pm 0.16 \mathrm{~g}$; curcumin: $3.92 \pm 0.17 \mathrm{~g}$ ) or weight gain (control chow: $3.51 \pm 0.49 \mathrm{~g}$ gain from day 1 ; curcumin chow: $3.52 \pm 0.30 \mathrm{~g}$ gain from day 1) over a 30-day period. Mice appeared to tolerate dietary curcumin well and showed no observable signs of distress.

\section{Chronic social defeat stress}

Screening. One week after arrival to the colony room, retired CD-1 breeders were screened for aggressive behavior for 3 consecutive days. They were individually housed in large plastic cages $(30.8 \mathrm{~cm} \times 30.8 \mathrm{~cm} \times 14.29 \mathrm{~cm}$ ) (Thoren Caging Systems, Hazleton, PA) modified to accommodate a divider, where they remained throughout the duration of social defeat stress. Social defeat began $24 \mathrm{~h}$ after the last day of screening.
Chronic social defeat stress. CSDS was performed as previously described [25]. Experimental mice were placed in the home cage of a CD-1 aggressor where they were allowed to interact for $5 \mathrm{~min}$. They were then placed behind a clear perforated Plexiglas divider within the aggressor's home cage for $24 \mathrm{~h}$, which prevented further physical contact, but allowed for continuous psychological stress from sensory cues. This procedure was repeated for ten consecutive days, with each experimental mouse being exposed to a novel CD-1 aggressor each day. Non-defeated control mice were housed the same way, but were never exposed to a CD-1 aggressor. Instead, they interacted with a novel mouse of the same strain each day and were housed with that mouse in the same cage for $24 \mathrm{~h}$, separated by a perforate divider. After the final defeat session, all mice were individually housed in standard mouse cages.

Social interaction test

Social interaction was tested as previously described [26]. Mice were placed in the middle of an open field arena $(25 \mathrm{~cm} \times 48 \mathrm{~cm})$ containing two identical wire-mesh enclosures for $5 \mathrm{~min}$. One enclosure contained a novel CD-1 mouse and the other was empty. Sessions were videotaped and an observer blind to treatment quantified the amount of time mice spent within each interaction zone, which was defined as the $12.5 \mathrm{~cm} \times 12.5 \mathrm{~cm}$ square region around each enclosure. A defeat index (DI) was calculated by dividing the difference in the time spent with each enclosure (CD-1 enclosure-empty enclosure) by total time with both enclosures. Mice with DI values greater than 0 were defined as "resilient" and mice with DI values below 0 were defined as "susceptible." Resilient mice on curcumin were called "responders" and susceptible mice on curcumin were called "non-responders."

Elevated plus maze

The apparatus consisted of two open arms and two arms enclosed by walls, all of which were connected by a central platform $50 \mathrm{~cm}$ above the ground (Stoelting, Wood Dale, IL). Mice were individually placed on the center platform facing an open arm and were given $5 \mathrm{~min}$ to explore the maze under low light conditions (70 lux). An overhead camera recorded behavior for later scoring by an observer blind to treatment group.

Open field (OF)

Mice were placed in the corner of a novel square chamber $(45 \times 45 \mathrm{~cm})(710 \mathrm{lux})$ and monitored for $10 \mathrm{~min}$. An overhead camera recorded all behavior, which was later analyzed using ANY-maze software (Stoelting, Wood Dale, IL) that tracked the animal and calculated total distance traveled $(\mathrm{cm})$, number of center entries, and time spent in the center half of the arena.

Acute restraint stress

Mice were individually restrained in a plastic DecapiCones (Braintree Scientific, Braintree, MA) secured with a binder clip for $15 \mathrm{~min}$.

Plasma IL-6 and corticosterone measurements

Trunk blood was collected in $1.5 \mathrm{ml}$ tubes containing $5 \mu \mathrm{l} 0.5 \mathrm{M}$ EDTA within $4 \mathrm{~h}$ after the onset of the light cycle and immediately placed on ice. Samples were centrifuged $(10 \mathrm{~min}$ at $835 \times g)$ and the supernatant was transferred to fresh tubes and stored at $-20^{\circ} \mathrm{C}$ until assay. Plasma IL-6 and corticosterone levels were determined with ELISA kits (Catalog number: ADI-900-045 (IL-6); ADI-900-097 (corticosterone); Enzo Life Sciences, Farmingdale, NY) according to the manufacturer's instructions.

\section{Brain IL-6 measurements}

Whole brains were removed immediately after the social interaction test, frozen with dry ice, and stored at $-80^{\circ} \mathrm{C}$. 
RNA extraction and $q R T-P C R$ analysis. Tissue punches from the nucleus accumbens and ventral hippocampus were flash frozen for subsequent processing. RNA was extracted with Trizol (Thermofisher, MA) and purified using the RNeasy Micro kit (Qiagen, MD) following the manufacturer's protocol. RNA was reverse transcribed with qScript cDNA Supermix (Quanta Bio, MA) and quantitative reverse transcription polymerase chain reaction (qRT-PCR) was performed with SYBR Green Mastermix (Quanta Bio, MA) using a QuantStudio 7 qRT-PCR system at the Epigenetics Core Facility at the Advanced Science Research Center, CUNY.

Protein. Bilateral 14-gauge punches of the nucleus accumbens and ventral hippocampus were collected from $1 \mathrm{~mm}$ coronal slices of frozen brains at $-20^{\circ} \mathrm{C}$ and stored at $-80^{\circ} \mathrm{C}$. Punches were later homogenized in ice-cold PIPES buffer (100 mM PIPES, $500 \mathrm{mM} \mathrm{NaCl}, 0.2 \%$ Triton X-100, 0.1\% $\mathrm{NaN}_{3}, 2 \mathrm{mM}$ EDTA, $5 \mu \mathrm{g} / \mathrm{mL}$ aprotinin, $0.1 \mu \mathrm{g} / \mathrm{mL}$ pepstatin $\mathrm{A}, \mathrm{pH}$ 7.0) and centrifuged at $15,000 \times g$ for $30 \mathrm{~min}$. Supernatant was collected and protein concentration was determined by bicinchoninic acid protein assay (ThermoFisher, MA). IL-6 protein levels were analyzed with an ELISA kit (Invitrogen, CA) according to manufacturer's instructions.

Statistical analyses

Statistical analyses were carried out with GraphPad prism software (GraphPad, San Diego) and significance levels were set at $p<0.05$. Data were analyzed with Bonferroni corrected $t$ tests and two- or three-way ANOVA. Interactions were followed by planned Student's $t$ post hoc tests with the Bonferroni adjustment.

\section{RESULTS}

129/SvEv mice demonstrate enhanced CSDS-induced social avoidance compared to C57BL/6J mice

The 129/SvEv strain exhibits higher levels of anxiety-like behavior than the C57BL/6 strain [27]. Although it has been suggested that they are more susceptible to CSDS than C57BL/6 mice [28], a direct comparison between the two strains using a standardized protocol has not been made. We tested the effects of CSDS in a large cohort of $129 / \mathrm{SvEv}$ mice $(n=50)$ and found that $92 \%$ of defeated mice avoid the CD-1 in the social interaction test. Using the same CSDS protocol, we found that only $33 \%$ of C57BL/6 mice avoided the social target, indicating that the 129/SvEv strain is significantly more susceptible to this type of chronic stressor $\left(x^{2}(1)=28.3, p<0.0001\right.$ ) (Fig. 1b, d). A two-way ANOVA on interaction time in defeated mice revealed a strain $\times$ object interaction $\left(F_{(1,72)}=83.75, p<0.0001\right)$. Defeated 129s spent more time with the empty enclosure and less time with the CD-1 than defeated C57s ( $p<0.0001$ for each post hoc comparison). Withingroup comparisons revealed that defeated $129 \mathrm{~s}$ spent less time with the CD-1 than the empty enclosure $(p<0.0001)$, while defeated C57s spent approximately the same amount of time with both enclosures (Fig. 1c).

\section{Dietary curcumin during CSDS prevents development of social} avoidance

We next tested whether dietary curcumin promotes resilience to CSDS in 129/SvEv mice (Fig. 2a). Of the mice that were defeated, a significantly higher proportion of curcumin-fed mice (64\%) exhibited resilience to stress relative to mice fed control chow $(14 \%)$, as defined by DI scores above 0 in the social interaction test $\left(x^{2}(1)=7.985, p=0.004\right)$ (Fig. 2b, c). Furthermore, a three-way ANOVA on interaction time revealed a significant diet $\times$ stress $\times$ object interaction $\left(F_{(1,52)}=50.11, p<0.0001\right)$. Post hoc comparisons revealed that defeated mice on a curcumin diet spent more time with the CD-1 $(p<0.001)$ and less time with the empty enclosure $(p<0.001)$ than defeated mice on control chow (Fig. $2 d$, f). Interestingly, there were no differences between non-stressed mice on curcumin and non-stressed mice on control chow in the social interaction test, indicating that curcumin does not affect social behavior in the absence of stress (Fig. 2d).

Additional analyses of mice fed curcumin during CSDS revealed two distinct responses to the diet. We categorized curcumin-fed mice that exhibited resilience as "responders" and curcuminfed mice that were susceptible as "non-responders." A comparison of time spent with each enclosure revealed that responders spent more time with the CD-1 $\left(t_{(13)}=6.22, p<0.001\right)$ and less time with the empty enclosure $\left(t_{(13)}=4.02, p=0.001\right)$ than nonresponders (Fig. 2e). Although curcumin did not block the effects of stress on social interaction in all mice, it did lead to a 4.5 -fold increase in the proportion of mice that were resilient to stress (control chow: $14 \%$ vs. curcumin: $64 \%$ ).

Dietary curcumin attenuates stress-induced increases in peripheral and central IL-6

Previous work has demonstrated that CSDS-induced increases in the inflammatory cytokine IL- 6 contribute to the development of social avoidance [9]. We investigated whether curcumin regulates this inflammatory response to stress by measuring IL- 6 in the periphery and brain (Fig. 3a). Plasma collected immediately after the social interaction test confirmed that CSDS increased circulating levels of IL-6 (main effect of stress: $F_{(1,36)}=29.68$, $p<0.0001$ ) and that curcumin attenuated this response (stress $X$ diet interaction: $\left.F_{(1,36)}=6.30, p=0.01\right)$. Post hoc comparisons revealed that defeated mice on control chow had significantly more plasma IL- 6 than non-defeated mice on control chow $(p<0.0001)$ and defeated mice on curcumin $(p<0.01$; Fig. $3 b)$. An analysis of defeated mice on curcumin revealed that curcumin prevented upregulation of peripheral IL- 6 only in responders $\left(t_{(11)}=3.54, p<0.01\right.$, Fig. $\left.3 c\right)$. There was also a significant negative correlation between stress-induced increases in IL- 6 and DI scores of defeated mice ( $r=-0.61, p<0.001$; Fig. 3d).

To assay the effects of curcumin on cytokine expression in the brain, we measured IL- 6 mRNA and protein in the nucleus accumbens (NAc) and ventral hippocampus (VHPC), both of which have previously been shown to play a role in CSDS-induced social avoidance [6]. An analysis of IL-6 transcript levels revealed no main effect of stress (NAc: $F_{(1,27)}=2.38, p>0.05$; vHPC: $F_{(1,30)}=1.41$, $p>0.05$ ) and no stress $\times$ diet interaction (NAc: $F_{(1,27)}=0.141$, $p>0.05$; vHPC: $F_{(1,30)}=0.357, p>0.05$; Fig. $3 e$, i) in either brain region. An analysis of defeated mice on curcumin revealed no difference between responders and non-responders in IL-6 mRNA in the NAc $\left(t_{(7)}=0.30, p>0.05\right)$ or $\operatorname{vHPC}\left(t_{(9)}=0.66, p>0.05\right)$ (Figs. 3f, j). However, our analysis of IL-6 protein levels in the NAc revealed a significant stress $\times$ diet interaction $\left(F_{(1,32)}=6.569\right.$, $p=0.01$ ), with more IL-6 protein in defeated mice on control chow than non-defeated mice on control chow $(p<0.05)$ and defeated mice on curcumin $(p=0.056$; Fig. $3 g)$. Curcumin responders and nonresponders had similarly low levels of IL- 6 protein in the NAc $\left(t_{(9)}=1.35, p>0.05\right.$; Fig. $\left.3 \mathrm{~h}\right)$. In contrast, our analysis of IL-6 protein in the $\mathrm{VHPC}$ revealed no significant main effect of stress $\left(F_{(1,33)}=0.035, p>0.05\right)$, no significant stress $\times$ diet interaction $\left(F_{(1,33)}>0.05\right.$; Fig. $\left.3 \mathrm{k}\right)$ and no significant difference between curcumin responders and non-responders $\left(t_{(10)}=0.35, p>0.05\right.$; Fig. 3l). Together these results indicate that curcumin may promote resilience by preventing stress-induced production of IL- 6 in the periphery and possibly the NAc.

Curcumin blocks stress-induced increases in HPA axis activation in responders

Previous studies have demonstrated that chronic stress alters sensitivity of the HPA axis [29]. We tested whether curcumin prevents the effects of CSDS on later activation of the HPA axis by measuring plasma corticosterone at two time points: (1) immediately after the social interaction test and (2) immediately after $15 \mathrm{~min}$ of restraint stress (Fig. 4a). We found that levels of corticosterone were similarly low following the social interaction 
A

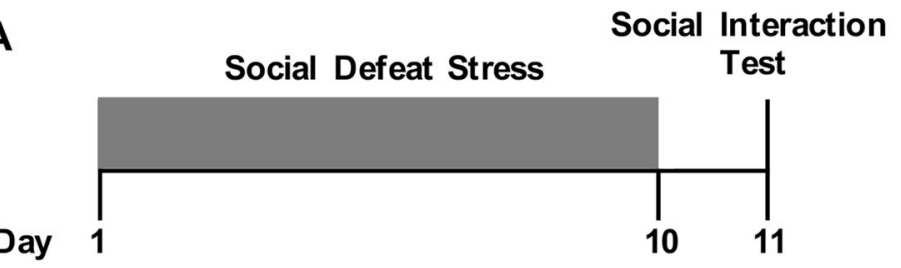

B
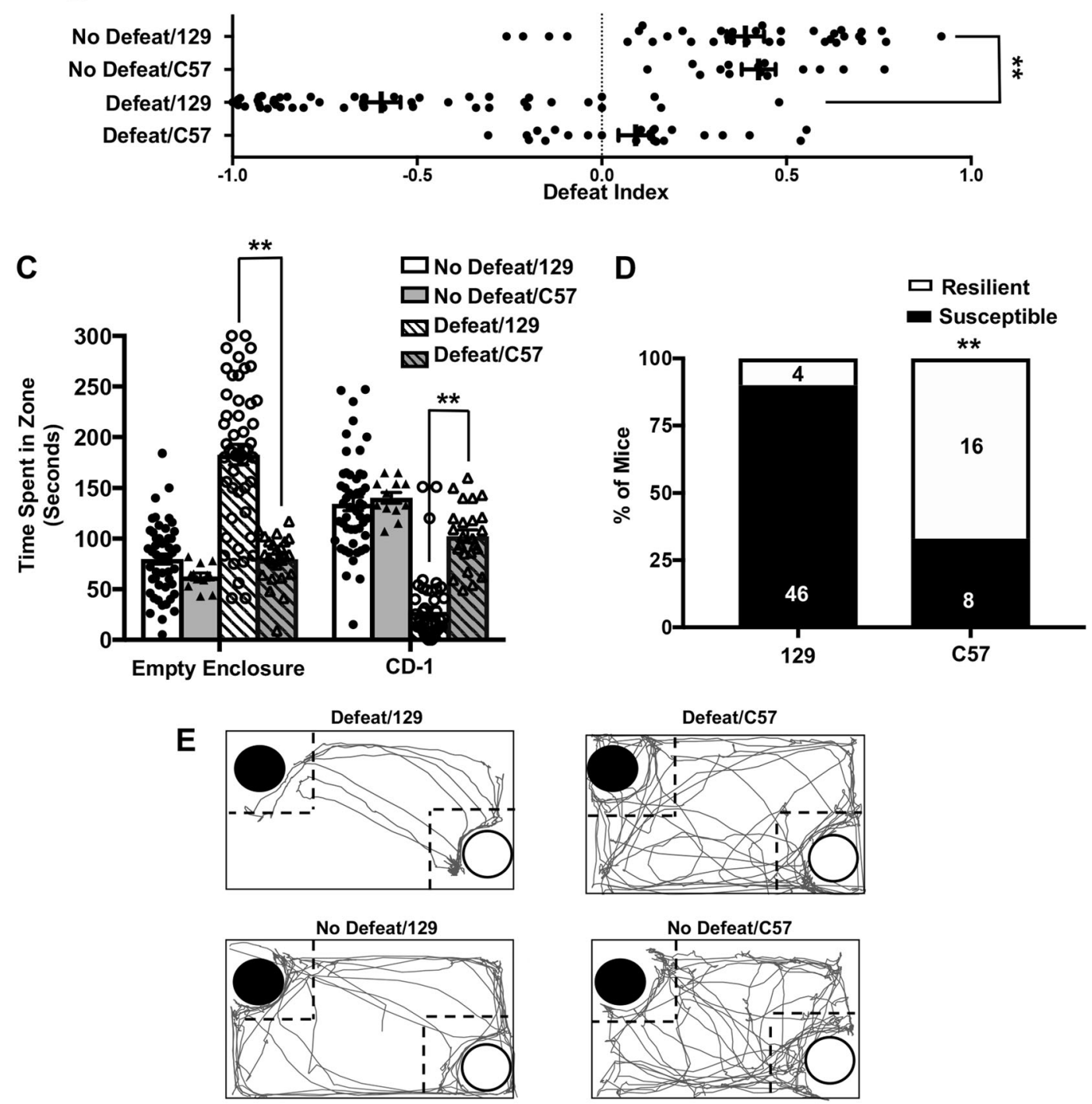

Fig. 1 129/SvEv mice are more susceptible to CSDS than C57BL/6J mice. a Schematic of behavioral procedures. b Distribution of DI scores for control and defeated mice of each strain. Mice with DI scores below 0 and were categorized as susceptible. c Time spent with the empty enclosure and the enclosure containing a novel CD-1. Defeated 129Sv/Ev mice spent significantly more time with the empty enclosure and less time with the CD-1 than non-defeated mice of the same strain and defeated C57BL/6J mice. Data represent mean \pm SEM. d Percent of defeated mice of each strain categorized as resilient or susceptible. Number of animals is indicated inside the bar graph. $\mathbf{e}$ The path (purple) of a representative mouse from each group during the social interaction test (black circle indicates CD-1; white circle indicates empty enclosure; dotted-line indicates interaction zone). No defeat/129 $(n=50)$, no defeat/C57 $(n=12)$, defeat/129 $(n=50)$, and defeat C57 ( $n=24)$. ${ }^{* *} p<0.0001 ; \wedge \wedge p<0.0001$ vs. defeat $/ 129$

test in all groups (main effect of stress: $F_{(1,36)}=1.25, p>0.05$; main effect of diet: $F_{(1,36)}=0.82, p>0.05$; stress $\times$ diet interaction: $F_{(1,36)}=0.12, p>0.05$; treatment response: $\left.t_{(8)}=0.48, p>0.05\right)$ (Fig. 4b, c). There was also no correlation between corticosterone levels measured after the social interaction test and DI scores $(r=0.26, p>0.05)$ (Fig. 4d). However, exposure to acute restraint stress 11 days after the last defeat session led to higher levels of circulating corticosterone in defeated animals, regardless of diet (main effect of stress: $F_{(1,36)}=17.56, p<0.001$ ) (Fig. 4e). An analysis of defeated mice on curcumin revealed that this upregulation of corticosterone was restricted to the animals characterized as non-responders based on their social interaction scores $\left(t_{(8)}=2.93, p=0.01\right)$ (Fig. $\left.4 f\right)$. Consistent with this finding, there was a significant negative correlation between restraintinduced increases in corticosterone and DI scores $(r=-0.66$, $p<0.01$ ) (Fig. 4g). Together, these results demonstrate that 

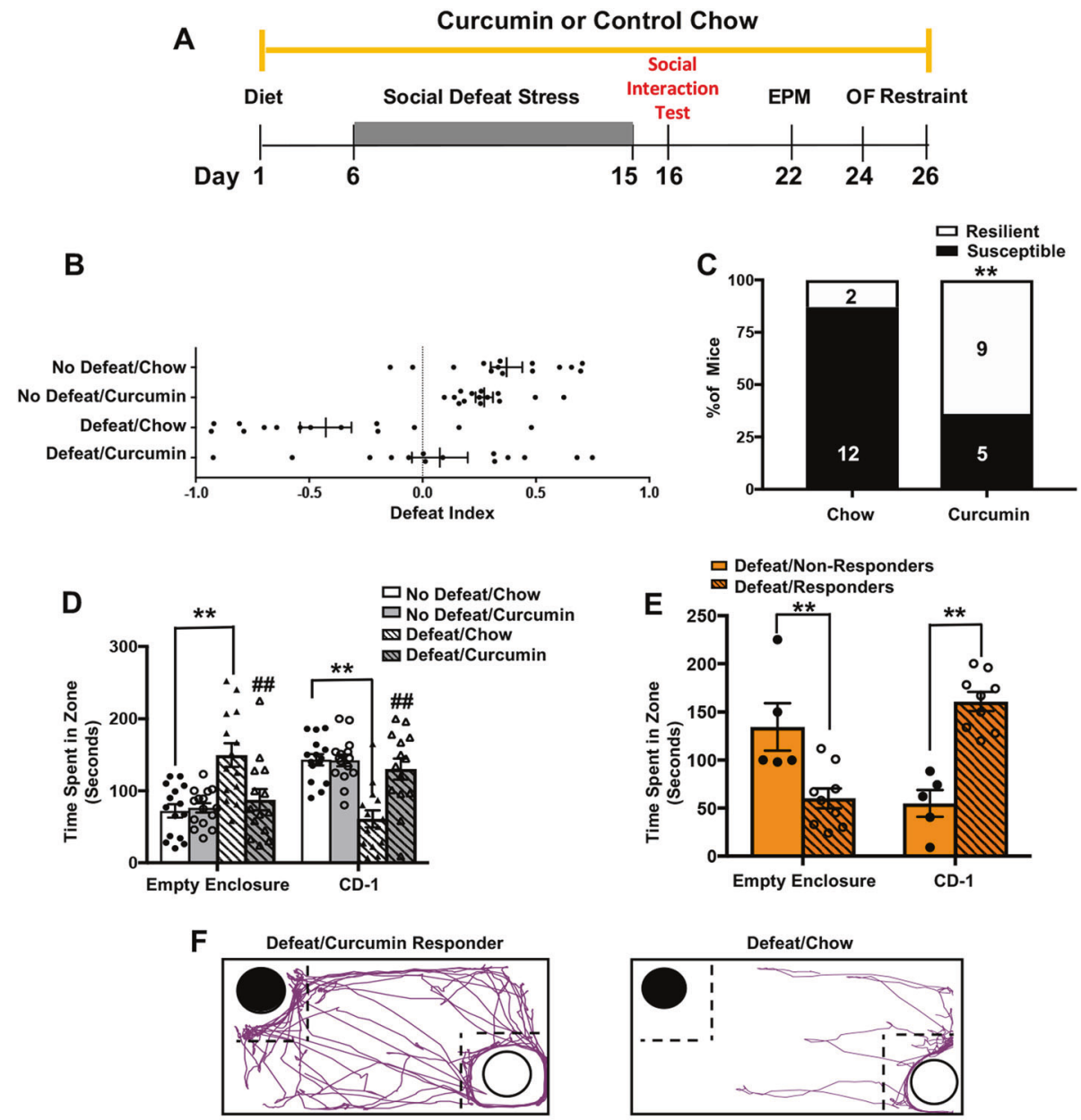

No Defeat/Curcumin
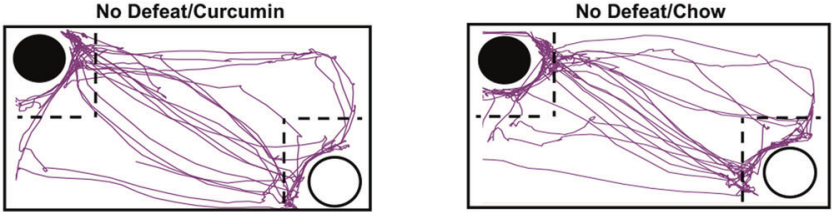

Fig. 2 Dietary curcumin promotes resilience to CSDS. a Schematic of behavioral procedures. b Distribution of DI scores for defeated and nondefeated $129 / \mathrm{SvEv}$ mice fed dietary curcumin or control chow. There were more defeated mice on curcumin than defeated mice on control chow that were categorized as resilient ( $\mathrm{DI}>0$ ). c Percent of defeated mice on each chow (control chow or curcumin) that were categorized as resilient or susceptible. Number of animals is indicated inside the bar graph. $\mathbf{d}$ Time spent with the empty enclosure and the enclosure containing a novel CD-1. Defeated mice on control chow spent more time with the empty enclosure and less time with the CD-1 than defeated mice on curcumin ( $p<0.0001$ for each comparison) and non-defeated mice on control chow ( $p<0.0001$ for each comparison). e Time that defeated mice on curcumin spent with either the empty enclosure or the enclosure containing a novel CD-1. Mice that were curcumin responders ( $\mathrm{DI}>0 ; n=9)$ spent significantly less time with the empty enclosure $(p=0.001)$ and more time with the CD-1 $(p=0.0001)$ than curcumin non-responders $(\mathrm{DI}<0 ; n=5)$. $\mathrm{f}$ The path (purple) of a representative mouse from each group during the social interaction test (black circle indicates CD-1; white circle indicates empty enclosure; dotted-line indicates interaction zone). Data represent mean \pm SEM. No defeat/chow $(n=14)$, no defeat/curcumin $(n=14)$, defeat/chow $(n=14)$, defeat/curcumin $(n=14) .{ }^{* *} p<0.0001 ;{ }^{\# \#} p<0.0001$ vs. defeat/chow. Defeat/responders $(n=5)$, defeat/non-responders $(n=5)$

response to curcumin in the social interaction test predicts the magnitude of later activation of the HPA axis by an acute stressor.

Dietary curcumin during CSDS prevents the development of anxiety-like behavior

We next investigated whether dietary curcumin prevents the lasting effects of CSDS on the expression of anxiety-like behavior. Mice were evaluated in two well-established tests of anxiety, the elevated-plus maze and the open field test, starting one week after the last social defeat session (Fig. 5a). Consistent with previous studies in this strain [26], we found that defeated mice on a control chow spent less time in open arms and more time in the closed arms of the elevated-plus maze than non-stressed mice on the same diet. A comparison across groups revealed a significant stress $\times$ diet interaction $\left(F_{(1,52)}=14.73, p<0.001\right)$ for time spent in the open arms (Fig. 5b) and time spent in the closed arms $\left(F_{(1,52)}=6.583, p<0.05\right)$ (Fig. $\left.5 c\right)$. Importantly, defeated mice on curcumin spent more time in the open arms $(p<0.0001)$ and less time in the closed arms $(p<0.05)$ than defeated mice on control chow, indicating that curcumin prevented stress-induced anxiety-like behavior. Interestingly, animals categorized as responders and non-responders based on social interaction scores did not differ in time spent in open or closed arms (main effect of treatment response: $\left.F_{(1,26)}=1.17, p>0.05\right)$ (Fig. $5 \mathrm{~d}$ ). 
A

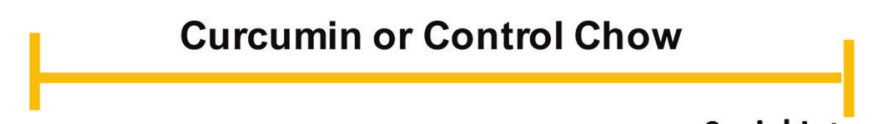

Diet Social Defeat Stress

Social Interaction

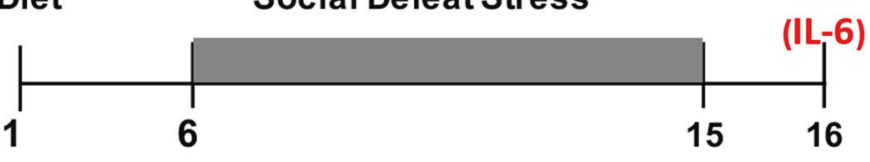

Day 1

6

Plasma
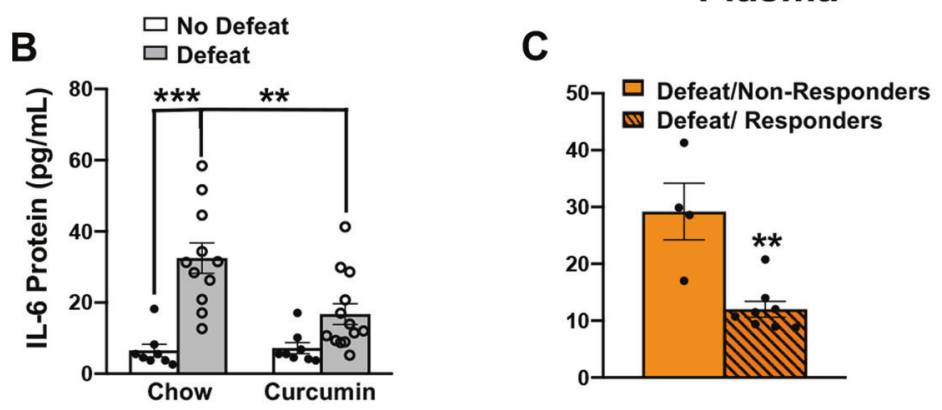

D
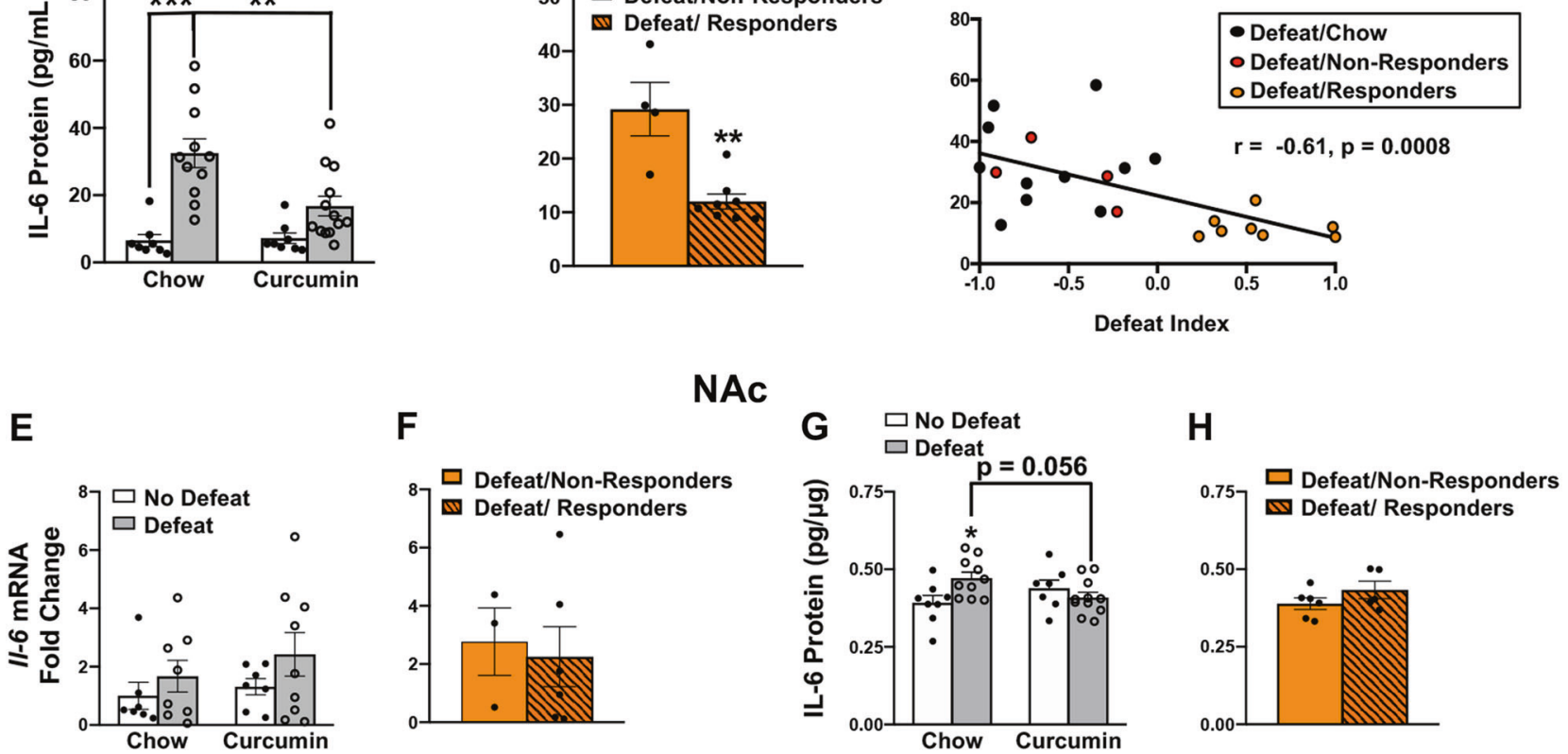

vHPC

I

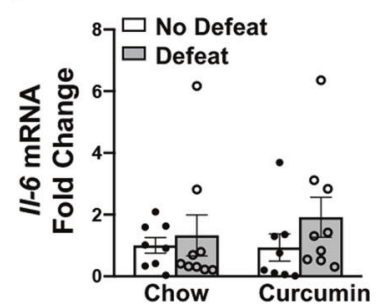

J

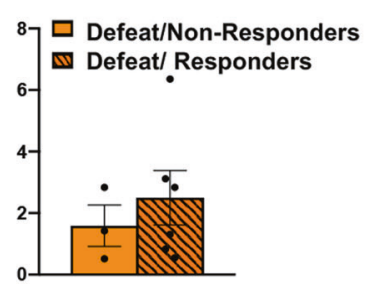

K

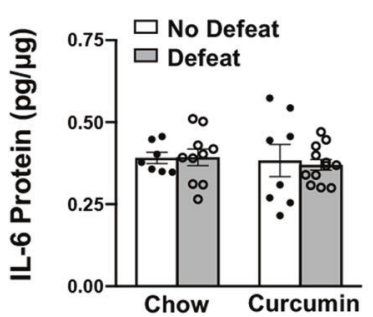

L

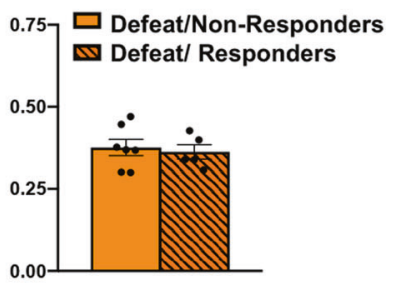

Fig. 3 Dietary curcumin attenuates CSDS-induced production of IL-6. a Schematic of behavioral procedures. b, c Plasma IL-6 collected immediately following the social interaction test. b Defeated mice on control chow had significantly more plasma IL- 6 than non-defeated mice on control chow $(p<0.0001)$ and defeated mice on curcumin $(p=0.001)$. c Curcumin responders (DI $>0)$ had significantly less plasma IL- 6 than curcumin non-responders $(\mathrm{DI}<0)$. d Scatterplot showing a significant negative correlation between DI scores and IL- 6 levels in the plasma of defeated mice $(r=-0.61, p<0.001)$. e, $\mathbf{f} \mathrm{IL}-6 \mathrm{mRNA}$ in the NAc expressed as fold change relative to non-defeated mice on control chow. $\mathbf{g}, \mathbf{h}$ IL-6 protein in the NAc. g Defeated mice on control chow had significantly higher levels of IL-6 protein in the NAc than non-defeated mice on control chow $(p<0.05)$ and defeated mice on curcumin $(p=0.056)$. h Curcumin responders and non-responders had similar levels of IL-6 protein in the NAc. i, $\mathbf{j}$ IL- 6 mRNA in the vHPC expressed as fold change relative to non-defeated mice on control chow. $\mathbf{k}$, I IL- 6 protein in the vHPC. No significant group differences were detected in the vHPC. Data represent mean \pm SEM. No defeat/chow $(n=9-11)$, no defeat/curcumin $(n=8-11)$, defeat/chow $(n=8-13)$, defeat/curcumin $(n=8-13) .{ }^{*} p<0.05$ vs. no defeat/chow, ${ }^{* *} p<0.01,{ }^{* * *} p<0.0001$. Defeat/responders $(n=3-7)$, defeat/non-responders $(n=5-7)$

We found similar effects of curcumin when animals were evaluated in the open field test. An analysis of time and entries into the center of the arena each revealed significant stress $\times$ diet interactions (time: $F_{(1,36)}=5.57, p<0.05$; entries: $F_{(1,36)}=6.79$, $p<0.05$ ) (Fig. 5e, f). Within the defeated group, mice on curcumin spent more time in the center $(p<0.05)$ and entered the center more often $(p<0.05)$ than mice on control chow. Similar to what we found in the elevated-plus maze, there were no differences observed in open field behavior between curcumin responders and non-responders (time: $t_{(8)}=1.2, p>0.05$; entries: $t_{(8)}=0.56$, 


\section{Curcumin or Control Chow}

A

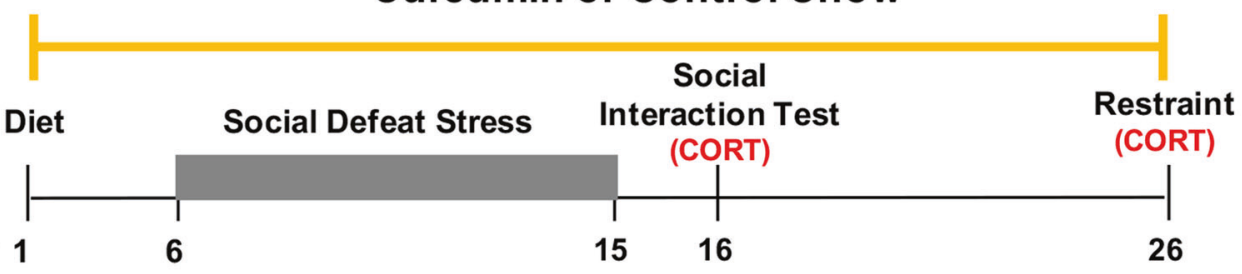

B
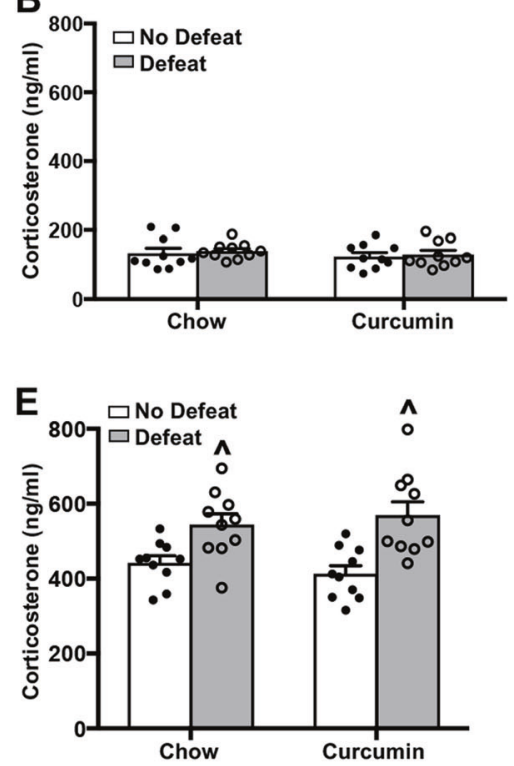

C

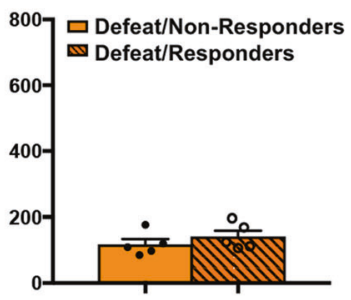

F $\square$ Defeat/Non-Responders

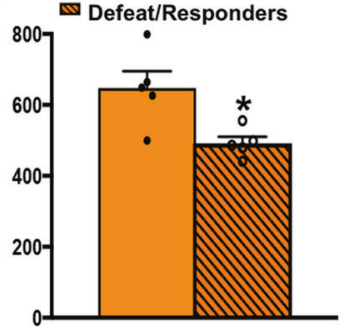

D

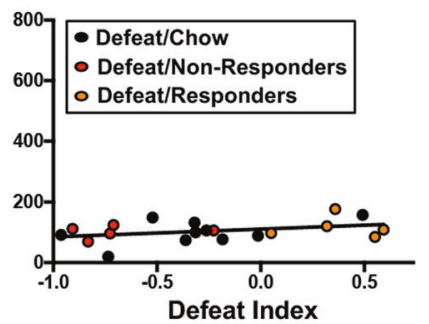

$\mathbf{G}$

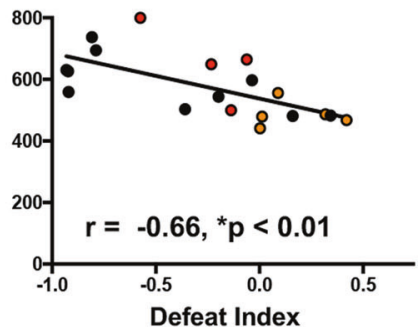

Fig. 4 Curcumin blocks stress-induced increases in HPA axis activation in responders. a Schematic of behavioral procedures. $\mathbf{b}$, $\mathbf{c}$ Plasma corticosterone collected immediately following the social interaction test. $\mathbf{d}$ Scatterplot showing that there was no correlation between DI scores and corticosterone levels in defeated mice. No defeat/chow $(n=10)$; no defeat/curcumin $(n=10)$; defeat/chow $(n=10)$; defeat/ curcumin ( $n=10)$; defeat/responders $(n=5)$; defeat/non-responders $(n=5)$. e, $\mathbf{f}$ Plasma corticosterone collected immediately following 15 min of restraint stress. Defeated mice had higher levels of corticosterone, regardless of diet. Curcumin responders (DI $>0)$ had lower levels of corticosterone than curcumin non-responders $(\mathrm{DI}<0)(p<0.05)$. g Scatterplot showing a significant negative correlation between $\mathrm{DI}$ scores and corticosterone levels in defeated mice $(r=-0.65, p<0.01)$. No defeat/chow $(n=10)$, no defeat/curcumin $(n=10)$, defeat chow $(n=10)$, defeat/curcumin $(n=10)$, defeat/responders $(n=5)$, and defeat/non-responders $(n=5)$. Data represent mean \pm SEM. ${ }^{*} p<0.05, \wedge p<0.05$ vs. no defeat group on the same diet

$p>0.05$ ) (Fig. 5h, i). An analysis of total distance traveled revealed no significant group differences (main effect of stress: $F_{(1,36)}=1.27, p>0.05$; main effect of diet: $F_{(1,36)}=0.32, p>0.05$; stress $\times$ diet interaction: $F_{(1,36)}=1.82, p>0.05$ ) (Fig. $5 \mathrm{~g}$ ) and no significant effect of treatment response $\left(t_{(8)}=0.98, p>0.05\right)$ (Fig. $5 \mathrm{j}$ ), indicating that the behavioral changes we reported with curcumin are not attributable to differences in overall movement. Together, these results indicate that curcumin administered during stress exposure prevents the development of anxiety-like behavior, which is independent of the effects of curcumin on social interaction.

Dietary curcumin administered after CSDS does not rescue the effects of prior stress exposure

In our previous experiments, curcumin administration began prior to CSDS and continued throughout the duration of the experiment. As such, the behavioral effects we report could be attributed to a prophylactic effect of curcumin or an ability of curcumin to rescue the effects of prior stress exposure. To test the later possibility, we ran a new experiment in which curcumin was administered after CSDS and mice were tested in the same series of behavioral tests used in our previous experiments (Figure S1A). CSDS-induced social avoidance was tested prior to treatment (stress $\times$ object interaction: $F_{(1,38)}=179.02, p<0.0001$; Figure S1B) so that mice later allocated to curcumin and control chow conditions would have similarly low levels of social interaction $(p>0.05)$. Treatment began immediately after the first social interaction test. We found no effect of curcumin on social interaction after two weeks of treatment (diet $\times$ stress $\times$ object interaction: $F_{(1,38)}=0.60, p>0.05$; Figure S1C) or 3 weeks of treatment (diet $\times$ stress $\times$ object interaction: $F_{(1,38)}=0.05, p>0.05$; Figure S1D). A comparison of the amount of time each defeated mouse spent with the CD-1 before and after 3 weeks of treatment revealed no significant difference between mice on the curcumin or control chow diet $\left(t_{(24)}=0.359, p>0.05\right.$; Figure S1E), confirming that dietary curcumin administered after CSDS does not reverse stress-induced social avoidance. In the elevated-plus maze, there was a significant main effect of stress for time spent in the open $\left(F_{(1,38)}=4.51, p<0.05\right)$ and closed arms $\left(F_{(1,38)}=5.12, p<0.05\right)$. However, there was no significant interaction in either the open (stress $\times$ diet interaction: $F_{(1,38)}=0.21, p>0.05$ ) or closed arms (stress $\times$ diet interaction: $F_{(1,38)}=0.01407, p>0.05$ ), indicating that curcumin administered after CSDS does not reverse anxiety-like behavior (Figure S2B, C). In the open field test, there was no significant main effect of stress for time in the center $\left(F_{(1,38)}=0.76\right.$, $p>0.05)$; Figure S2D) or entries into the center $\left(F_{(1,38)}=0.34\right.$, $p>0.05$; Figure S2E). While there was a near significant stress $X$ diet interaction for time $\left(F_{(1,38)}=4.10, p=0.05\right)$ and a significant 


\section{Curcumin or Control Chow}

A
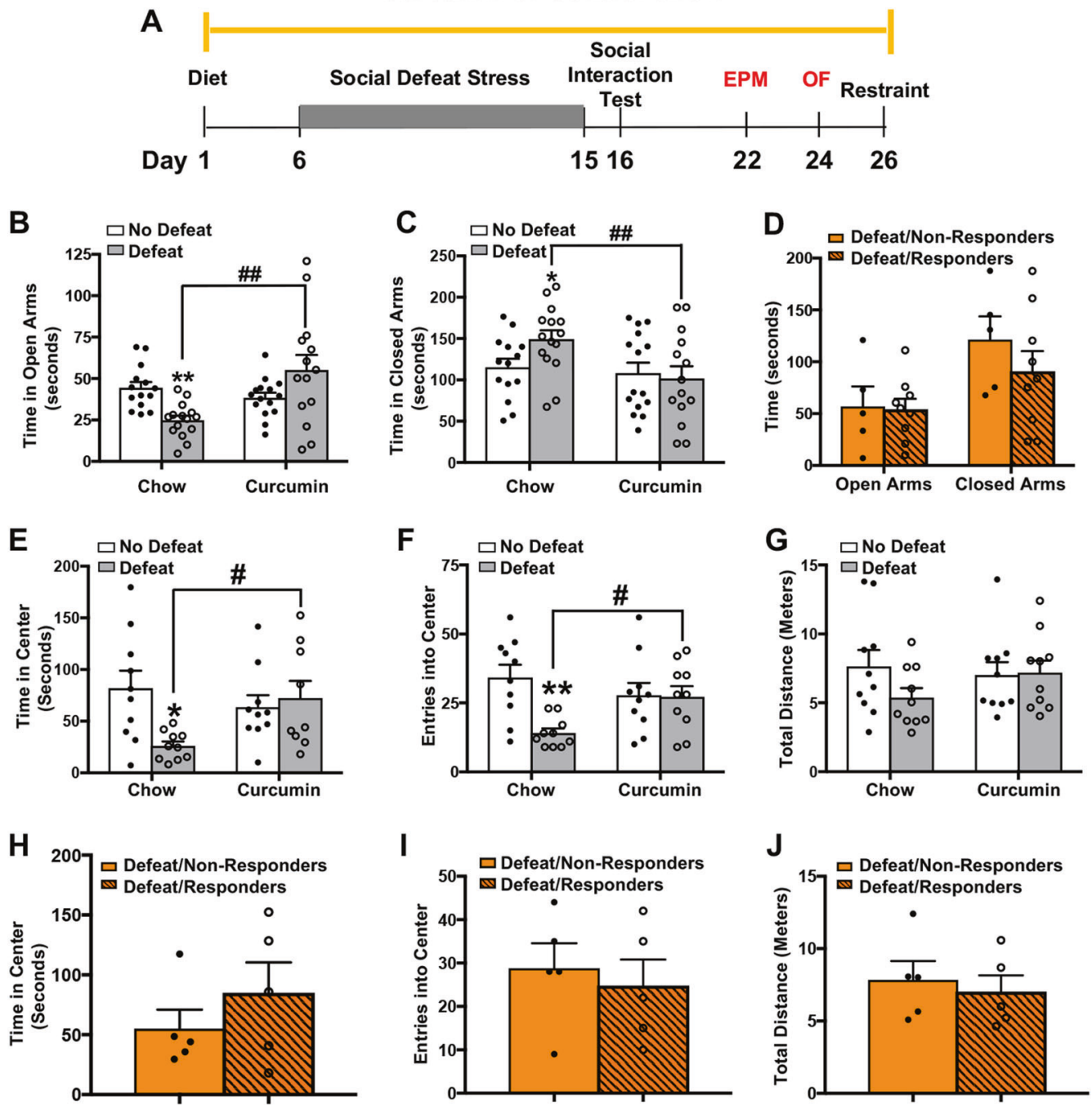

Fig. 5 Dietary curcumin protects against the anxiety-like effects of CSDS in the EPM and OF. a Schematic of behavioral procedures. $\mathbf{b}, \mathbf{c}$ Defeated mice on control chow spent less time in the open arms and more time in the closed arms of the EPM than non-defeated mice on control chow and defeated mice on curcumin. $\mathbf{d}$ Curcumin responders ( $\mathrm{DI}>0$ ) and non-responders $(\mathrm{DI}<0)$ did not differ in the amount of time spent in the open or closed arms of the EPM. e, $\mathbf{f}$ Defeated mice on control chow spent less time and made fewer entries into the center of the OF than non-stressed mice on control chow and defeated mice on curcumin. $\mathbf{g}$ Groups did not differ in total distance traveled. $\mathbf{h}-\mathbf{j}$ There were no significant differences between curcumin responders and non-responders in time spent in the center, entries into the center, or total distance traveled in the OF. Data represent mean \pm SEM. For EPM: no defeat/chow $(n=14)$, no defeat/curcumin $(n=14)$, defeat/ chow $(n=14)$, defeat/curcumin $(n=14)$, defeat/responders $(n=9)$, and defeat/non-responders $(n=5)$. ${ }^{* *} p<0.0001$ vs. no defeat/chow, ${ }^{*} p<0.05$ vs. no defeat/chow, ${ }^{\# \#} p<0.001$. For OF: no defeat/chow $(n=10)$, no defeat/curcumin $(n=10)$, defeat/chow $(n=10)$, defeat/curcumin $(n=10)$, defeat/responders $(n=5)$, defeat/non-responders $(n=5) .{ }^{* *} p<0.001$ vs. no defeat/chow, ${ }^{*} p<0.01$ vs. no defeat/chow, ${ }^{\#} p<0.05$

interaction for entries into the center of the OF $\left(F_{(1,38)}=4.25\right.$, $p<0.05)$, none of the post hoc tests reached significance. When mice were exposed to acute restraint stress 27 days after CSDS, we found no significant main effect of stress on plasma corticosterone $\left(F_{(1,16)}=0.84, p>0.05\right)$ and no stress $\times$ diet interaction $\left(F_{(1,16)}=0.08, p>0.05\right.$; Figure S2F).

\section{DISCUSSION}

Promoting resilience to stress may prevent the development of stress-induced psychiatric disorders, but resilience-enhancing compounds are not yet clinically available. Using CSDS, we show that a diet enriched with curcumin produces a 4.5 -fold increase in resilience when administered during stress exposure to $129 / \mathrm{SvEv}$ mice, which are highly susceptible to this type of stress. Although the overall effects of curcumin are striking, we identified two distinct responses to treatment based on social avoidance (responders and nonresponders). Interestingly a positive response to curcumin in the social interaction test is associated with reduced activation of the immune system and the HPA axis. Specifically, curcumin responders have lower levels of the cytokine IL-6 in the periphery and release less corticosterone following a novel acute stressor than non-responders. Importantly, curcumin blocks the development of anxiety-like behavior in both responders and non-responders in two well-established preclinical tests of anxiety [30]. Interestingly, we find that curcumin administered after CSDS does not rescue these effects of stress. Collectively, our findings provide the first preclinical evidence that curcumin promotes resilience to CSDS and may prevent social avoidance and other symptoms of anxiety if given to individuals during exposure to chronic stress.

Curcumin is a biologically active polyphenol compound found in the rhizome of the turmeric plant (Curcuma longa), which is commonly used as a spice in Indian and East Asian cuisines. 
Although turmeric contains several different curcuminoids, the therapeutic effects of curcumin (diferuloylmethane) have been the most widely studied, with doses that range from $0.5-1.5 \mathrm{~g} / \mathrm{day}$ in humans. Curcumin has been shown to effectively prevent or treat several diseases with an inflammatory component, including rheumatoid arthritis, ulcerative colitis and type 2 diabetes [15-17, 32]. Recent double-blind placebo-controlled clinical trials indicate that curcumin reduces symptoms of depression, with effects that are comparable to selective serotonin reuptake inhibitors [19, 20, 33]. Although to our knowledge curcumin has not been tested in patients with anxiety disorders, it has been found to improve State and Trait scores in the Spielberger State-Trait Anxiety Inventory (STAI) in people with major depressive disorder [19]. It has also been shown in one study to block the anxiogenic effects of acute restraint stress in the elevated-plus maze in rats [34]. Others show that curcumin impairs the formation of fear memories in rats in the absence of stress [23]. Like the study reported here, these preclinical studies administered curcumin prior to experimental manipulation, indicating that curcumin may be a promising therapeutic option for reducing fear and anxiety in patients if it is administered as a prophylactic.

Given that many of the diseases mentioned above have an inflammatory component, the anti-inflammatory properties of curcumin may be central in mediating its therapeutic effects. Curcumin is best known for inhibiting the IKK-NFKB signaling pathway, which is integral in regulating inflammatory processes and activating the immune response $[35,38]$. Importantly, CSDS activates the IKK-NFKB signaling pathway in the periphery and brain, which in turn plays an essential role in the development of stress-induced depression- and anxiety-like behavior [39, 40]. For example, upregulation of the downstream cytokine IL-6 in the periphery has been shown to be necessary for CSDS-induced social avoidance behavior [9]. Our finding that dietary curcumin attenuates CSDSinduced upregulation of IL-6 in the periphery and nucleus accumbens indicates that curcumin may promote resilience by targeting this signaling pathway. Although curcumin has been shown to decrease the production of IL-6 in previous cell culture studies [41], this is the first study to demonstrate that curcumin prevents IL- 6 production in response to stress. Interestingly, the effect of stress on IL- 6 and the attenuation of this effect by curcumin were much more pronounced in the periphery than the brain, indicating that the periphery may be an initial site of action. Orally administered curcumin does accumulate at high levels within the gastrointestinal tract $[42,43]$ and in vitro studies show that curcumin protects against inflammation-induced degradation of intestinal epithelial cells [44]. It is therefore possible that curcumin prevents deterioration of the lining of the intestines during exposure to psychosocial stress, which would prevent bacteria from translocating into systemic circulation. This, in turn, would prevent activation of the transcription factor NF-KB and downstream pro-inflammatory cytokines, such as IL-6. While there is evidence that a "leaky gut" is clinically relevant [45], future studies are required to test whether dietary curcumin promotes resilience by blocking an inflammatory response initiated by the gut.

Similar to the heterogeneous response to antidepressant treatment found in human populations, we found two distinct treatment responses to curcumin based on social avoidance behavior. Unlike non-responders, responders failed to show stressinduced upregulation of the cytokine IL-6 in the periphery, indicating that treatment response may result from differential effects of curcumin on the NF-KB pathway. Further evidence implicating the involvement of the IKK-NFKB pathway in treatment response is found in clinical studies showing that the antidepressant effects of ketamine and SSRIs are associated with a decrease in plasma IL-6, which was not found in patients who were characterized as treatment resistant $[46,47]$. Although our results support a role of this inflammatory pathway in mediating resilience in curcumin responders, it is still unclear why some animals did not respond. Future studies would benefit from characterizing the baseline differences between responders and nonresponders at the behavioral and molecular level. Such findings may provide insight into which clinical populations would benefit most from curcumin treatment.

Interestingly, the effects of curcumin on social avoidance did not predict treatment response in tests of anxiety. That is, both responders and non-responders displayed the same decrease in anxiety in the elevated-plus maze and open field test. This is consistent with previous studies showing that C57BL/6J mice characterized as resilient or susceptible based on their social interaction behavior did not differ when tested in the elevatedplus maze [5]. CSDS-induced social avoidance resembles the social withdrawl that is commonly reported in depressed patients and is often interpreted as a measure of depressive-like behavior $[5,48-50]$. Our data indicate a dissociation between the effects of curcumin on this stress-induced depressive-like behavior and more typical measures of anxiety-like behavior.

\section{CONCLUSIONS}

In summary, we provide strong evidence that dietary curcumin promotes resilience to chronic stress. Our findings suggest that treatment with curcumin may be a safe and effective therapeutic agent for preventing the development of social withdrawal and other symptoms of anxiety in individuals who are highly susceptible to stress, if it is administered during stress exposure. Future preclinical studies focused on characterizing differences between responders and non-responders may provide insight into the clinical populations that would benefit most from curcumin treatment.

\section{FUNDING AND DISCLOSURE}

This work was supported by funding from the US National Institute on Minority Health and Health Disparities of the $\mathrm{NIH}$ (G12MD007599) to NSB and GES, a PSC-CUNY Award to NSB, and a PSC-CUNY Award to J.L. We thank the Epigenetics Core Facility in the CUNY Advanced Science Research Center.

\section{ADDITIONAL INFORMATION}

Supplementary Information accompanies this paper at (https://doi.org/10.1038/ s41386-018-0295-2)

Conflict of interest: The authors declare that they have no conflict of interest.

Publisher's note: Springer Nature remains neutral with regard to jurisdictional claims in published maps and institutional affiliations.

\section{REFERENCES}

1. Kessler RC, Petukhova M, Sampson NA, Zaslavsky AM, Wittchen HU. Twelve-month and lifetime prevalence and lifetime morbid risk of anxiety and mood disorders in the United States. Int J Methods Psychiatr Res. 2012;21:169-84

2. Kendler KS, Karkowski LM, Prescott CA. Causal relationship between stressful life events and the onset of major depression. Am J Psychiatry. 1999;156:837-41.

3. Köhler CA, Evangelou E, Stubbs B, Solmi M, Veronese N, et al. Mapping risk factors for depression across the lifespan: An umbrella review of evidence from metaanalyses and Mendelian randomization studies. J Psychiatr Res 2018;5:1-9.

4. Bagot RC, Cates HM, Purushothaman I, Lorsch ZS, Walker DM, et al. Circuit-wide transcriptional profiling reveals brain region-specific gene networks regulating depression susceptibility. Neuron. 2016:90:969-83.

5. Krishnan V, Han MH, Graham DL, Berton O, Renthal W, et al. Molecular adaptations underlying susceptibility and resistance to social defeat in brain reward regions. Cell. 2007;131:391-404.

6. Bagot RC, Parise EM, Pena CJ, Zhang HX, Maze I, et al. Ventral hippocampal afferents to the nucleus accumbens regulate susceptibility to depression. Nat Commun. 2015;6:7062. 
7. Chuang J-C, Krishnan V, Hana GY, Mason B, Cui H, et al. A $\beta 3$-adrenergic-leptinmelanocortin circuit regulates behavioral and metabolic changes induced by chronic stress. Biol Psychiatry. 2010a;67:1075-82.

8. Chuang JC, Cui H, Mason BL, Mahgoub M, Bookout AL, et al. Chronic social defeat stress disrupts regulation of lipid synthesis. J Lipid Res. 2010b;51:1344-53.

9. Hodes GE, Pfau ML, Leboeuf M, Golden SA, Christoffel DJ, et al. Individual differences in the peripheral immune system promote resilience versus susceptibility to social stress. Proc Natl Acad Sci USA. 2014;111:16136-41.

10. Chaudhury D, Walsh JJ, Friedman AK, Juarez B, Ku SM, et al. Rapid regulation of depression-related behaviours by control of midbrain dopamine neurons. Nature. 2013;493:532.

11. Donahue RJ, Muschamp JW, Russo SJ, Nestler EJ, Carlezon WA. Effects of striatal $\triangle$ FosB overexpression and ketamine on social defeat stress-induced anhedonia in mice. Biol Psychiatry. 2014;76:550-58.

12. Duvoix A, Blasius $R$, Delhalle $S$, Schnekenburger $M$, Morceau $F$, et al. Chemopreventive and therapeutic effects of curcumin. Cancer Lett. 2005; 223:181-90.

13. Xu Y, Lin D, Li S, Li G, Shyamala SG, et al. Curcumin reverses impaired cognition and neuronal plasticity induced by chronic stress. Neuropharmacology. 2009;57:463-71.

14. Jobin C, Bradham CA, Russo MP, Juma B, Narula AS, Brenner DA, et al. Curcumin blocks cytokine-mediated NF-KB activation and proinflammatory gene expression by inhibiting inhibitory factor I-KB kinase activity. J Immunol. 1999;163:3474-83.

15. Chuengsamarn S, Rattanamongkolgul S, Luechapudiporn R, Phisalaphong C, Jirawatnotai S. Curcumin extract for prevention of type 2 diabetes. Diabetes Care. 2012;35:2121-27.

16. Daily JW, Yang M, Park S. Efficacy of turmeric extracts and curcumin for alleviating the symptoms of joint arthritis: a systematic review and meta-analysis of randomized clinical trials. J Med Food. 2016;19:717-29.

17. Hanai $H$, lida $T$, Takeuchi $K$, Watanabe $F$, Maruyama $Y$, et al. Curcumin maintenance therapy for ulcerative colitis: randomized, multicenter, double-blind, placebo-controlled trial. Clin Gastroenterol Hepatol. 2006;4:1502-06.

18. McFadden R-MT, Larmonier CB, Shehab KW, Midura-Kiela M, Ramalingam R, et al. The role of curcumin in modulating colonic microbiota during colitis and colon cancer prevention. Inflamm Bowel Dis. 2015;21:2483-94.

19. Lopresti AL, Drummond PD. Efficacy of curcumin, and a saffron/curcumin combination for the treatment of major depression: a randomised, double-blind, placebo-controlled study. J Affect Disord. 2017;207:188-96.

20. Lopresti AL, Maes M, Maker GL, Hood SD, Drummond PD. Curcumin for the treatment of major depression: a randomised, double-blind, placebo controlled study. J Affect Disord. 2014;167:368-75.

21. Hurley LL, Akinfiresoye L, Nwulia E, Kamiya A, Kulkarni AA, Tizabi Y. Antidepressantlike effects of curcumin in WKY rat model of depression is associated with an increase in hippocampal BDNF. Behav Brain Res. 2013;239:27-30.

22. Xu Y, Ku B, Tie L, Yao H, Jiang W, et al. Curcumin reverses the effects of chronic stress on behavior, the HPA axis, BDNF expression and phosphorylation of CREB. Brain Res. 2006;1122:56-64.

23. Monsey MS, Gerhard DM, Boyle LM, Briones MA, Seligsohn M, Schafe GE. A diet enriched with curcumin impairs newly acquired and reactivated fear memories. Neuropsychopharmacology. 2015;40:1278-88.

24. Prasad S, Tyagi AK, Aggarwal BB. Recent developments in delivery, bioavailability, absorption and metabolism of curcumin: the golden pigment from golden spice. Cancer Res Treat. 2014b;46:2-18.

25. Golden SA, Covington HE III, Berton O, Russo SJ. A standardized protocol for repeated social defeat stress in mice. Nat Protoc. 2011;6:1183-91.

26. Brachman RA, McGowan JC, Perusini JN, Lim SC, Pham TH, et al. Ketamine as a prophylactic against stress-induced depressive-like behavior. Biol Psychiatry. 2016;79:776-86.

27. Holmes A, Wrenn C, Harris A, Thayer K, Crawley J. Behavioral profiles of inbred strains on novel olfactory, spatial and emotional tests for reference memory in mice. Genes Brain Behav. 2002;1:55-69.

28. Dadomo H, Sanghez V, Di Cristo L, Lori A, Ceresini G, Malinge I, et al. Vulnerability to chronic subordination stress-induced depression-like disorders in adult 129SvEv male mice. Prog Neuropsychopharmacol Biol Psychiatry. 2011;35:1461-71.
29. Herman JP. Neural control of chronic stress adaptation. Front Behav Neurosci. 2013;7:61.

30. Carola V, D'Olimpio F, Brunamonti E, Mangia F, Renzi P. Evaluation of the elevated plus-maze and open-field tests for the assessment of anxiety-related behaviour in inbred mice. Behav Brain Res. 2002;134:49-57.

31. Chandran B, Goel A. A randomized, pilot study to assess the efficacy and safety of curcumin in patients with active rheumatoid arthritis. Phytother Res. 2012; 26:1719-25.

32. Irving GR, et al. Prolonged biologically active colonic tissue levels of curcumin achieved after oral administration-a clinical pilot study including assessment of patient acceptability. Cancer Prev Res. 2013;6:119-128.

33. Sanmukhani J, Satodia V, Trivedi J, Patel T, Tiwari D, et al. Efficacy and safety of curcumin in major depressive disorder: a randomized controlled trial. Phytother Res. 2014;28:579-85

34. Haider S, Naqvi F, Batool Z, Tabassum S, Sadir S, et al. Pretreatment with curcumin attenuates anxiety while strengthens memory performance after one short stress experience in male rats. Brain Res Bull. 2015;115:1-8.

35. Kopp EB, Ghosh S. NF-kB and Rel proteins in innate immunity. Adv Immunol. 1995;58:241-249.

36. Christoffel DJ, Golden SA, Dumitriu D, Robison AJ, Janssen WG, et al. IKB kinase regulates social defeat stress-induced synaptic and behavioral plasticity. J Neurosci. 2011:31:314-21.

37. Christoffel DJ, Golden SA, Heshmati M, Graham A, Birnbaum S, et al. Effects of inhibitor of $\mathrm{KB}$ kinase activity in the nucleus accumbens on emotional behavior. Neuropsychopharmacology. 2012;37:2615

38. Bhatt D, Ghosh S. Regulation of the NF-kB-mediated transcription of inflammatory genes. Front Immunol. 2014;5:1-9.

39. Jain SK, Rains J, Croad J, Larson B, Jones K. Curcumin supplementation lowers TNF-a, IL-6, IL-8, and MCP-1 secretion in high glucose-treated cultured monocytes and blood levels of TNF-a, IL-6, MCP-1, glucose, and glycosylated hemoglobin in diabetic rats. Antioxid Redox Signal. 2009;11:241-249.

40. Irving GR, et al. Prolonged biologically active colonic tissue levels of curcumin achieved after oral administration-a clinical pilot study including assessment of patient acceptability. Cancer Prev Res. 2013;6:119-128.

41. Irving GR, et al. Prolonged biologically active colonic tissue levels of curcumin achieved after oral administration-a clinical pilot study including assessment of patient acceptability. Cancer Prev Res. 2013;6:119-128.

42. Irving GR, et al. Prolonged biologically active colonic tissue levels of curcumin achieved after oral administration-a clinical pilot study including assessment of patient acceptability. Cancer Prev Res. 2013;6:119-128.

43. Lopresti AL. The problem of curcumin and its bioavailability: could its gastrointestinal influence contribute to its overall health-enhancing effects? Adv Nutr. 2018;9:41-50.

44. Ma TY, Iwamoto GK, Hoa NT, Akotia V, Pedram A, Boivin MA, et al. TNF-a-induced increase in intestinal epithelial tight junction permeability requires NF-KB activation. Am J Physiol Gastrointest Liver Physiol. 2004;286:G367-G376.

45. Kelly JR, Kennedy PJ, Cryan JF, Dinan TG, Clarke G, Hyland NP. Breaking down the barriers: the gut microbiome, intestinal permeability and stress-related psychiatric disorders. Front Cell Neurosci. 2015;9:392.

46. Kiraly D, Horn S, Van Dam N, Costi S, Schwartz J, et al. Altered peripheral immune profiles in treatment-resistant depression: response to ketamine and prediction of treatment outcome. Transl Psychiatry. 2017;7:e1065.

47. Yoshimura R, Hori H, Ikenouchi-Sugita A, Umene-Nakano W, Ueda N, Nakamura J. Higher plasma interleukin-6 (IL-6) level is associated with SSRI-or SNRI-refractory depression. Progr Neuropsychopharmacol Biol Psychiatry. 2009;33:722-26.

48. Bagot RC, Cates HM, Purushothaman I, Vialou V, Heller EA, et al. Ketamine and imipramine reverse transcriptional signatures of susceptibility and induce resilience-specific gene expression profiles. Biol Psychiatry. 2017;81:285-95.

49. Nestler EJ, Hyman SE. Animal models of neuropsychiatric disorders. Nat Neurosci. 2010;13:1161.

50. Berton O, McClung CA, Dileone RJ, Krishnan V, Renthal W, et al. Essential role of BDNF in the mesolimbic dopamine pathway in social defeat stress. Science. 2006;311:864-8. 